\title{
Identificação de microbacias contendo pivôs centrais no município de Sorriso (MT): estudo contributivo à outorga do uso de água
}

Raphael Maia Aveiro Cessa ${ }^{1}$

Thiago de Souza Rizzi

\section{Resumo}

O objetivo deste trabalho foi identificar no município de Sorriso (MT) microbacias hidrográficas contendo pivôs centrais, subsidiando o referenciamento dos valores das vazões a serem outorgadas. A área em estudo foi o município de Sorriso no estado de Mato Grosso, situado entre as coordenadas geográficas Latitude $12^{\circ} 33^{\prime} 31^{\prime \prime}$ Sul e Longitude 5542' 51" Oeste. A delimitação das microbacias hidrográficas na área estudada deu-se por meio do modelo digital de elevação (MDE), o qual permitiu a obtenção da carta hipsométrica empregando curvas de nível equidistantes de $20 \mathrm{~m}$, permitindo análise da altimetria do terreno e das redes de drenagem do município de Sorriso. O MDE do referido município foi obtido por meio de imagens (SC-21-X-B, SC-21-X-C, SC-21-X-D, SC-21-Z-C e SD-21-X-A), com resolução espacial de 90 m advinda da Shuttle Radar Topography Mission e disponibilizada pela Empresa Brasileira de Pesquisa Agropecuária. Não foram estimados valores de vazões de captação de água por pivôs contidos nas mesmas microbaciais no município de Sorriso (MT) acima de $70 \%$ da $Q_{95}$ considerada.

Palavras-chave: Recursos hídricos. Drenagem. Vazões.

\section{Introdução}

Os conflitos existentes associados à utilização de água estão relacionados à multiciplicidade do seu uso e aumento da demanda (GARCIA, 2007). No Brasil, a maior demanda de consumo de água vem da agricultura, seguida pelo setor industrial e uso doméstico (MMA, 2005).

A bacia hidrográfica é uma área composta basicamente de um conjunto de superfícies vertentes e de uma rede de drenagem formada por cursos d'água que confluem até resultar em um leito único no exutório (SILVEIRA, 2001). Suas delimitações e informações fluviométricas podem subsidiar a adoção de políticas de uso dos recursos hídricos e o processo de outorga de água para projetos de irrigação, além de constituir diretriz para instituições de crédito agrícola (AMORIM et al., 2010).

A outorga para uso da água foi efetivada no país pela Lei 9.433/97, a qual instituiu a Política Nacional dos Recursos Hídricos. Segundo Garcia et al. (2007), é necessária a derivação ou captação da parcela da água de corpos hídricos superficiais ou aquíferos destinada ao abastecimento público, processos produtivos industriais ou agrícolas, lançamento em corpo de água, de esgotos e demais resíduos líquidos ou gasosos, tratados ou não, transporte ou disposição final de resíduos, aproveitamento para os potenciais hidrelétricos e outros usos, que alterem o regime na quantidade ou na qualidade de água existente em um corpo de água.

1 Instituto Federal de Educação, Ciência e Tecnologia de Brasília - Campus Planaltina, professor do Ensino Básico, Técnico e Tecnológico - Área Agronomia. raphael.cessa@ifb.edu.br. Rodovia BR 128, km 21, Zona Rural de Planaltina, Brasília, Distrito Federal, CEP: 73380900.

2 Instituto Federal de Educação, Ciência e Tecnologia do Mato Grosso - Campus Sorriso, engenheiro agrônomo. thiago.rizzi@srs.ifmt.edu.br. 
A outorga do uso da água de corpos hídricos de superfície em Mato Grosso é realizada pela Secretaria Estadual de Meio Ambiente (SEMA), baseada tecnicamente na Resolução n 27, de 09 de julho de 2009, do Conselho Estadual de Recursos Hídricos.

Na resolução citada anteriormente tem-se como critério o índice $Q_{95}$, que é a vazão de referência com garantia de permanência em 95 \% do tempo, considerando a bacia de contribuição no ponto de captação. Dessa forma, as outorgas das vazões com validade de médio e longo prazo poderão ser emitidas quando o limite máximo de derivações consuntivas for igual ou inferior a $50 \%$ do $Q_{95}$. Ainda consta na Resolução que a soma das vazões outorgadas na bacia não poderá exceder 70 \% da vazão de referência definida pelo $Q_{95}$.

A adequada gestão dos recursos hídricos é dada pelo conhecimento do comportamento hidrológico das bacias hidrográficas e, portanto, a definição da vazão outorgada é imprescindível a partir daquela de referência (ARNÉZ, 2002).

A determinação da disponibilidade hídrica necessita de monitoramento contínuo para criação de um banco de dados (série histórica) das vazões dos mananciais, representando o comportamento fluviométrico de uma bacia hidrográfica nem sempre disponível (FIOREZE et al., 2008). A maioria das bacias hidrográficas não dispõe de dados fluviométricos suficientes, contemplando, na maioria das vezes, grandes bacias hidrográficas, o que promove incertezas na avaliação quantitativa dos recursos hídricos (SILVEIRA; TUCCI, 1998).

Segundo a Empresa de Pesquisa Energética - EPE (2009), a produção de grãos de soja em todo estado de Mato Grosso tem êxito, principalmente pelo cultivo da planta na Bacia do Rio Teles Pires, nos municípios de Sorriso, Nova Mutum e Lucas do Rio Verde. Aproximadamente 70 \% do município de Sorriso é utilizado no cultivo de oleaginosas, amiláceas e/ou criação pecuária, sendo que 11.728,17 hectares são submetidos à irrigação por pivôs centrais (Figura 1) com vazão média de captação de águas superficiais de $0,20 \mathrm{~m}^{3} \mathrm{~s}^{-1}$ (estimativa com base nos valores disponíveis no Diário Oficial de Mato Grosso proveniente dos processos de autorização de outorga), possibilitando três safras em um único ano agrícola, com destaque para a "sequência" soja, milho e feijoeiro. 
Figura 1. Uso do solo no município de Sorriso no Estado de Mato Grosso

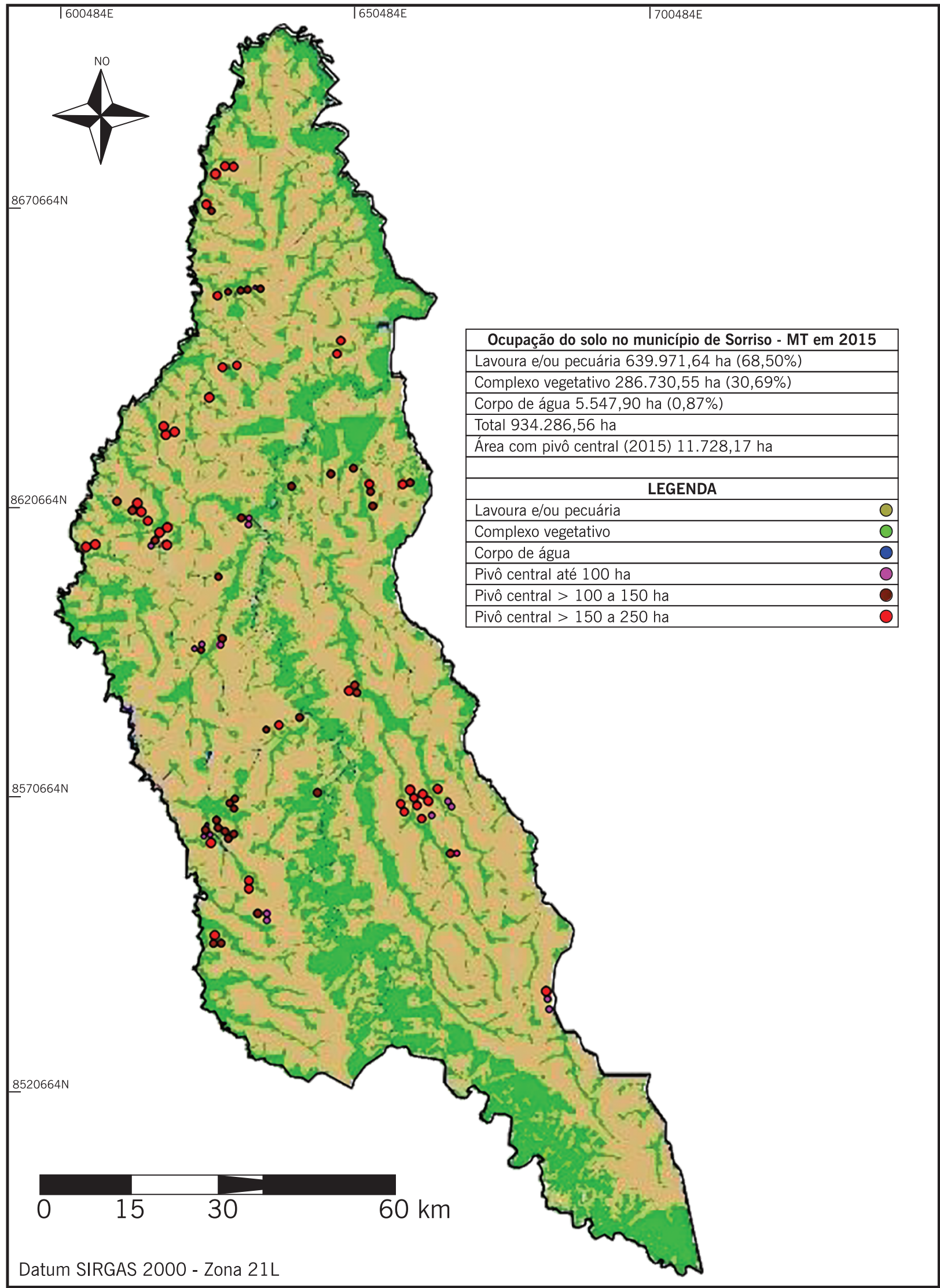

Fonte: Elaborada pelos autores. 
São 93 pivôs centrais localizados no município de Sorriso até o ano de 2015. Aproximadamente $18 \%$ têm áreas menores que 100 hectares e $80 \%$ com áreas maiores que 100 e até 200 hectares. Entre os anos de 2011 e 2015, houve aumento de pivôs centrais naquele município na ordem de 2,2 vezes.

O objetivo deste trabalho foi identificar no município de Sorriso (MT) microbacias hidrográficas contendo pivôs centrais, subsidiando o referenciamento dos valores das vazões a serem outorgadas.

\section{Material e métodos}

A área em estudo foi o município de Sorriso no Estado de Mato Grosso (Figura 2), situado entre

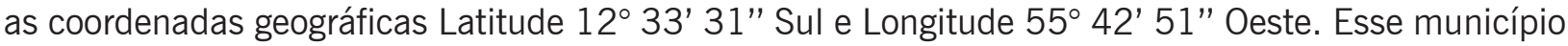
(9.329,6 km²) está contido na Bacia Hidrográfica Amazônica, Sub-bacia Hidrográfica do Tapajós, Microbacia do Alto do Rio Teles Pires.

Figura 2. Croqui de localização do município de Sorriso no Estado de Mato Grosso em bacias hidrográficas

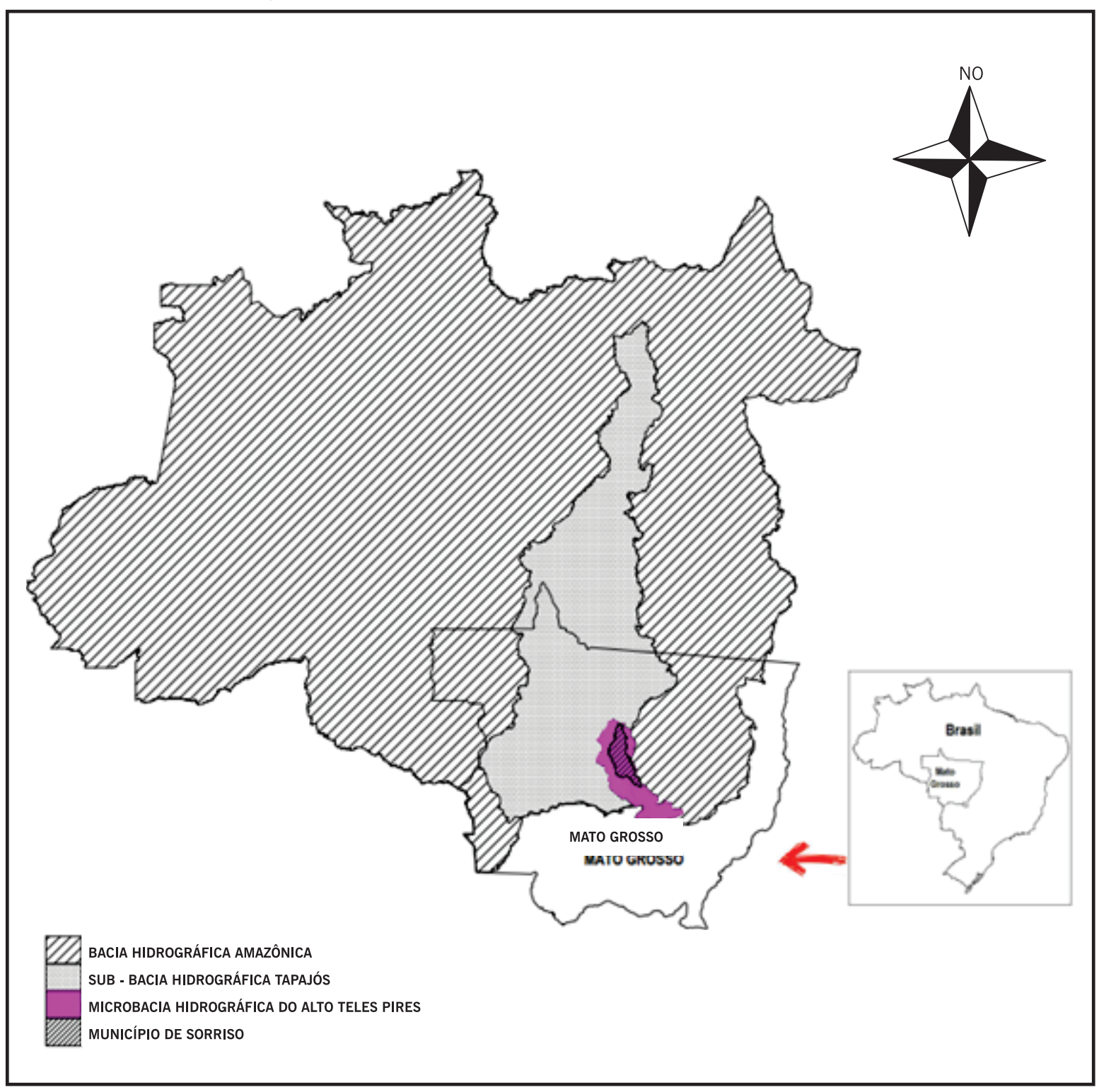

Fonte: Elaborada pelos autores. 
O mapa de uso de solo (Figura 1) foi obtido por meio da classificação das imagens de satélite LANSAT 5 em uma área delimitada a partir da Orbita 226, Ponto 69, com data de passagem registrada no dia 11 de novembro de 2015. Para tal, foram utilizadas as bandas 3(R) 4(G) 2(B), vermelho, verde e azul, respectivamente. Essa composição foi suficiente para detalhamento das características do solo e também para evidenciar a vegetação natural, exigidas no presente trabalho.

A extração de informação em imagens de satélite para reconhecer padrões e objetos homogêneos deu-se por meio de um classificador multiespectral "pixel a pixel" máxima verossimilhança (MAXVER), a qual utilizou apenas a informação espectral isoladamente de cada pixel para achar regiões homogêneas. A imagem foi dividida em conjunto de "pixels" contíguos que se espalham bidirecionalmente e que apresentam uniformidade formando regiões que devem corresponder às áreas de interesse da aplicação pela sua segmentação no processo de crescimento de regiões (agrupamento de dados na qual somente as regiões adjacentes, espacialmente, podem ser agrupadas) (PIZARRO et al., 2001), com valores de similaridades e área do pixel de $12 \mathrm{~m}$ x $15 \mathrm{~m}$.

A classificação da imagem deu-se pelo método semiautomático (supervisionado), com o classificador por região Bhattacharya. "O algoritmo utiliza a distância de Bhattacharya para medir a separabilidade estatística entre cada par de classe espectral" (LEÃO et al., 2007). A separabilidade é calculada por meio da distância média entre as distribuições de probabilidades de classes espectrais.

A delimitação das microbacias hidrográficas na área estudada deu-se segundo procedimentos descritos em Sperling (2007), por meio do modelo digital de elevação (MDE), o qual permitiu a obtenção da carta hipsométrica, empregando curvas de nível equidistantes de $20 \mathrm{~m}$, permitindo análise da altimetria do terreno e das redes de drenagem do município de Sorriso.

O MDE do referido município foi obtido por meio de imagens (SC-21-X-B, SC-21-X-C, SC-21-X-D, SC-21-Z-C e SD-21-X-A), com resolução espacial de 90 m advinda da Shuttle Radar Topography Mission e disponibilizada pela Empresa Brasileira de Pesquisa Agropecuária (MIRANDA, 2009), fazendo-se uso do aplicativo computacional SPRING 5.2.6.

Para efeito de discussão foi considerado o valor de $120,89 \mathrm{~m}^{3} \mathrm{~s}^{-1}$ para $\mathrm{Q}_{95}$ no exutório das microbacias identificadas contendo pivôs centrais no município de Sorriso (MT). Este valor foi estimado considerando-se ser $1 / 3$ do $Q_{95} 366,33 \mathrm{~m}^{3} \mathrm{~s}^{-1}$, valor este médio de vazão medido na estação fluviométrica Teles Pires, no município de Sorriso (MT), série histórica maio de 1976 a dezembro de 2009 extraído do trabalho de Veiga et al. (2013).

\section{Resultados e discussão}

Na Figura 3 pode-se observar a delimitação das microbacias contendo pivôs centrais no município de Sorriso. 
Figura 3. Microbacias contendo pivôs centrais no município de Sorriso no Estado de Mato Grosso

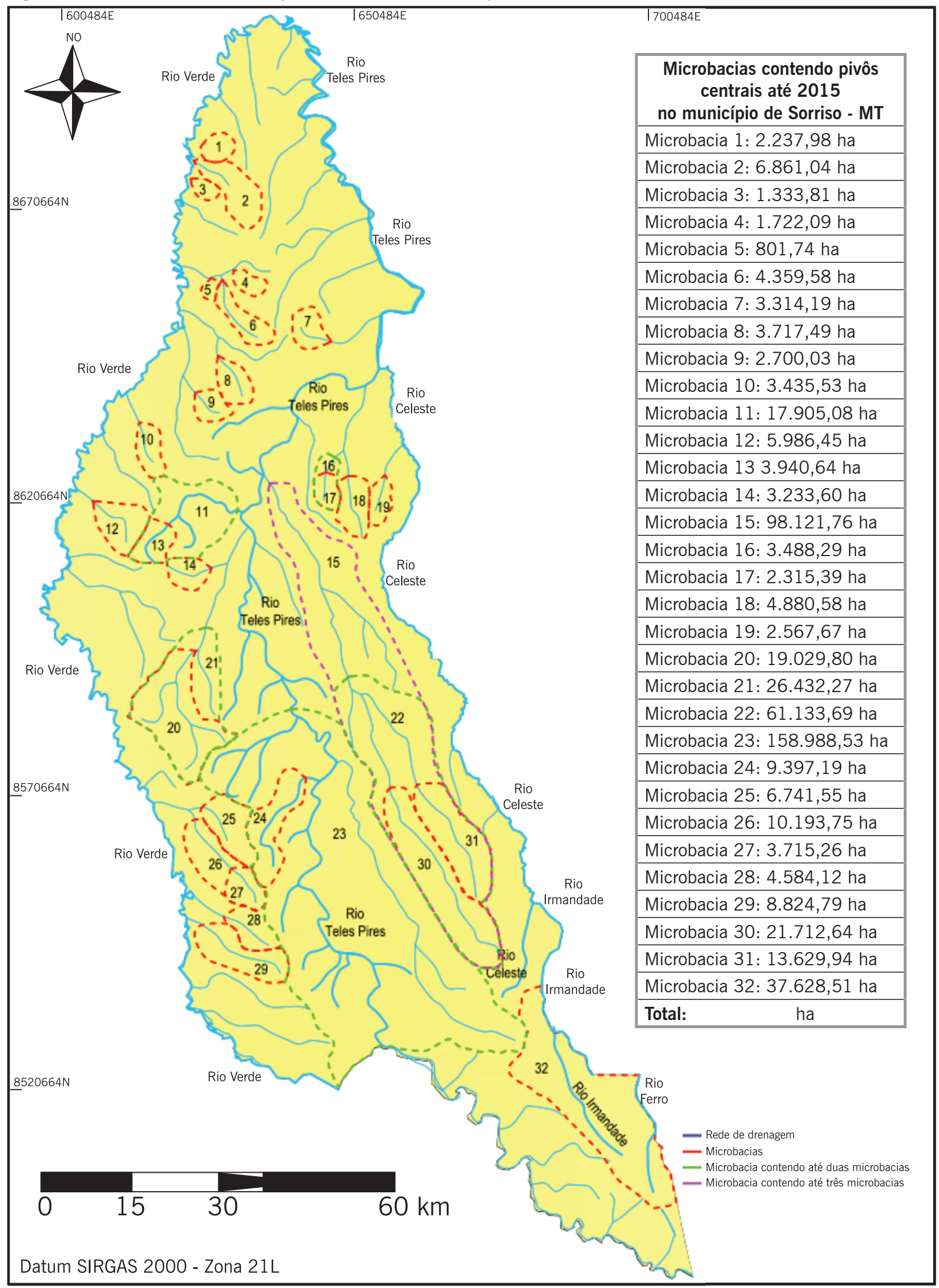

Fonte: Elaborada pelos autores. 
Para efeito de discussão, tendo como exemplo a microbacia 15 contendo 17 pivôs centrais com vazão média de captação de águas superficiais de $0,20 \mathrm{~m}^{3} \mathrm{~s}^{-1}$ (valor baseado no Diário Oficial de Mato Grosso proveniente dos processos de autorização de outorga), pode-se estimar uma captação volumétrica de água de $0,60 \mathrm{~m}^{3} \mathrm{~s}^{-1}$. Este valor é 25 vezes menor do que $70 \%$ do valor do $\mathrm{Q}_{95}$ $\left(120,89 \mathrm{~m}^{3} \mathrm{~s}^{-1}\right)$ no exutório daquela microbacia. Pelo mesmo raciocínio, as microbacias 11,21 , 23 e 29 teriam valores 42, 84, 60 e 140 vezes menores, respectivamente, observando os $70 \%$ do valor $\mathrm{Q}_{95} 120,89 \mathrm{~m}^{3} \mathrm{~s}^{-1}$.

No trabalho de Santos et al. (2015), referente à regionalização hidroclimatológica da Bacia Hidrográfica do Rio Tapajós, a vazão $Q_{95}$ obtida na estação fluviométrica na foz do Rio Teles Pires, no município de Aripuanã (MT) (série histórica de 1995 a 2009) foi de 1.106,00 $\mathrm{m}^{3} \mathrm{~s}^{-1}$, com média anual de $3.367,00 \mathrm{~m}^{3} \mathrm{~s}^{-1}$. A vazão média na Bacia do Alto Teles Pires, da qual o município de Sorriso ocupa aproximadamente $45 \%$, teve valor estimado de $843,00 \mathrm{~m}^{3} \mathrm{~s}^{-1}$ (EPE, 2009).

Embora as discussões realizadas no presente trabalho estejam baseadas em estimativas, devido à pouca disponibilidade de informações regionais sobre microbacias, bem como a complexidade na obtenção das séries históricas de vazões pelas inúmeras dificuldades (recurso financeiro, equipamentos apropriados, mão de obra em quantidade e especializada) de medições nos exutórios, nota-se que ainda é possível a emissão de outorgas de captação de água superficiais na maioria das microbacias hidrográficas que contém pivôs centrais no município de Sorriso (MT) pelas regras da SEMA, descritas na Resolução n 27, de 09 de julho de 2009, do Conselho Estadual de Recursos Hídricos. É imprescindível, portanto, que haja fiscalização operacional desses pivôs centrais pelos órgãos competentes, impondo horário escalonado de funcionamento e conferindo vazões "bombeadas".

\section{Conclusão}

De acordo com a metodologia utilizada no presente estudo, não foram estimados valores de vazões de captação de água por pivôs contidos nas mesmas microbaciais no município de Sorriso (MT) acima de $70 \%$ do $Q_{95}$ considerado.

\section{Identification of watersheds containing central pivots in the municipality of Sorriso (MT): a contributory study to the granting of water use}

The objective of this study was to identify watersheds containing central pivots in Sorriso (MT) municipality, supporting the flow rate value references to be granted. Sorriso is located in Mato Grosso State, in the following geographic coordinates Latitude $12^{\circ} 33^{\prime} 31^{\prime \prime}$ South and longitude $55^{\circ} 42$, 51" West. The delimitation of the watersheds in the studied area was given through the digital elevation model (DEM), which allowed the production of hipsometric letter using $20 \mathrm{~m}$ equidistant contour lines, allowing analysis of the ground altimetry and networks drainage of Sorriso. The DEM for Sorriso was obtained through images (SC-21-XB, SC-21-XC, SC-21-XD, SC-21-ZC and SD-21-XA), with a spatial resolution of $90 \mathrm{~m}$, from the Shuttle Radar Topography Mission and made available by The Brazilian Agricultural Research Corporation. It was not estimated amounts of water intake flow by pivots contained in the same watersheds in Sorriso (MT) municipality of over $70 \%$ of $Q_{95}$ considered.

Keywords: Water resources. Drainage. Streamflow. 


\section{Referências}

AMORIM, J. A. de; CRUZ, M. A. S.; RESENDE, R. S. Qualidade da água subterrânea para irrigação na bacia hidrográfica do Rio Piauí, em Sergipe. Revista Brasileira de Engenharia Agrícola e Ambiental, v. 14, n. 8, p. 804-811, 2010. Disponível em: <http://www.scielo.br/scielo.php?script=sci_arttext\&pid=S1415-43662010000800003>. Acesso em: 11 dez. 2017.

ARNÉZ, F. A. Análise de critérios de outorga do suo da água na bacia do rio Santa Maria, RS. 2002. 146 f. Dissertação (Mestrado em Engenharia de Recursos Hídricos e Saneamento Ambiental). Instituto de Pesquisas Hidráulicas, UFRGS, Porto Alegre. Disponível em: <http://www.lume.ufrgs.br/ handle/10183/1577> . Acesso em: 11 dez. 2017.

EMPRESA DE PESQUISA ENERGÉTICA (EPE). Avaliação ambiental integrada da bacia hidrográfica do rio Teles Pires: relatório final - sumário executivo. Disponível em: <http://www.epe.gov.br/MeioAmbiente/Documents/AAI\%20Teles\%20Pires/AAI\%20Teles\%20Pires\%20-\%20Relat\%C3\%B3rio\%20 Final\%20-\%20Sum\%C3\%A1rio\%20Executivo.pdf>. Acesso em: 07 abr. 2016.

FIOREZE, A. P.; OLIVEIRA, L. F. C.; FRANCO, A. P. B. Avaliação do desempenho de equações de regionalização de vazões na bacia hidrográfica do Ribeirão Santa Bárbara, Goiás, Brasil. Ambi-Água, v. 3, n. 2, p. 62-76, 2008. Disponível em: <http://www.ambi-agua.net/seer/index.php/ambi-agua/ article/viewFile/132/206 >. Acesso em: 11 dez. 2017.

GARCIA, A. V.; OLIVEIRA, E. C. A. de; SILVA, G. P.; COSTA, P. P. da; OLIVEIRA, L. A. de. Disponibilidade hídrica e volume de água outorgado na microbacia do Ribeirão Abóbora, município de Rio Verde, Estado de Goiás. Caminhos de Geografia, v. 8, n. 22, p. 97 - 106, 2007. Disponível em: $<$ http://www.seer.ufu.br/index.php/caminhosdegeografia/article/viewFile/15555/8804>. Acesso em: 11 dez. 2017.

LEÃO, C.; KRUG, L. A.; KAMPEL, M.; FONSECA, L. M. G. Avaliação de métodos de classificação em imagens TM/Landsat e CCD/CBERS para o mapeamento do uso e cobertura da terra na região costeira do extremo sul da Bahia. In: XIII Simpósio MMA (Ministério do Meio Ambiente). Consumo sustentável: manual de educação. Brasília: Consumers International, 2005. 160 p.

MIRANDA, E. E. de (Coord.). Brasil em Relevo. Campinas: Embrapa Monitoramento por Satélite, 2005. Disponível em: <http://www.relevobr.cnpm.embrapa.br>. Acesso em: 3 jun. 2016.

PIZARRO, P.; COMUNELLO E.; MANTELLI, S. Segmentação por Crescimento de Regiões. In: ALDO VON WANGENHEIM, Eds. Introdução à visão computacional. New York, Taylor e Francis, 2001. p. 35-64.

SANTOS, C. A. dos; ARAUJO, I. B. de; WANZELER, R. T. S.; SERRÃO, E. A. O.; FARIAS, M. H. C. S.; LIMA, A. M. M. de. Regionalização hidroclimatológica da Bacia Hidrográfica do Rio Tapajós. Revista Geografia Acadêmica, v. 9, n. 1, p. 32-51, 2015. Disponível em: < https://revista.ufrr.br/rga/article/ view/2929>. Acesso em: 11 dez. 2017.

SILVEIRA, A. L. L. Ciclo hidrológico e bacia hidrográfica. In: TUCCI, C.E.M. (Org.). Hidrologia: ciência e aplicação. São Paulo: EDUSP, 2001. p. 35-51. 
SILVEIRA, G. L.; TUCCI, C. E. M. Monitoramento em pequenas bacias para estimativa de disponibilidade hídrica. Revista Brasileira de Recursos Hídricos, v. 3, n. 3, p. 97-110, 1998. Disponível em: $<$ https://www.abrh.org.br/SGCv3/index.php?PUB=1\&ID=52\&SUMARIO=4334>. Acesso em: 11 dez. 2017.

SPERLING, M. V. Estudos e modelagem da qualidade da água de rios: princípios do tratamento biológico de águas residuárias. Belo Horizonte: UFMG, 2007. 196 p.

VEIGA, A. M.; MElO, D. C. R.; SOARES, A. K.; TRINDADE, M. C.; MELLO, L. T. A. de; SOUZA, R. M. de. Diagnóstico das vazões do Rio Teles Pires. XX Simpósio Brasileiro de Recursos Hídricos. In: XX Simpósio Brasileiro de Recursos Hídricos, 2013, Bento Gonçalves. XX Simpósio Brasileiro de Recursos Hídricos, 2013.

Histórico editorial:

Submetido em: 03/06/2016.

Aceito em: 13/09/2016.

Como citar:

ABNT

CESSA, R. M. A.; RIZZI, T. S. Identificação de microbacias contendo pivôs centrais no município de Sorriso (MT): estudo contributivo à outorga do uso de água. Revista Agrogeoambiental, Pouso Alegre, v. 9, n. 4, p. 23-31, out./dez. Doi: http://dx.doi.org/10.18406/2316-1817v9n420171009

APA

CESSA, R. M. A. \& RIZZI, T. S. (2017). Identificação de microbacias contendo pivôs centrais no município de Sorriso (MT): estudo contributivo à outorga do uso de água. Revista Agrogeoambiental, 9 (4), 23-31.

Doi: http://dx.doi.org/10.18406/2316-1817v9n420171009

ISO

CESSA, R. M. A. e RIZZI, T. S. Identificação de microbacias contendo pivôs centrais no município de Sorriso (MT): estudo contributivo à outorga do uso de água. Revista Agrogeoambiental, 2017, vol. 9, n. 4, pp. 23-31. Eissn 2316-1817. Doi: http://dx.doi.org/10.18406/2316-1817v9n420171009

VANCOUVER

Cessa RMA, Rizzi TS. Identificação de microbacias contendo pivôs centrais no município de Sorriso (MT): estudo contributivo à outorga do uso de água. Rev agrogeoambiental. 2017 out/dez; 9(4): 23-31.

Doi: http://dx.doi.org/10.18406/2316-1817v9n420171009 